\title{
NGTS-6b: an ultrashort period hot-Jupiter orbiting an old K dwarf
}

Jose I. Vines, ${ }^{1 \star}$ James S. Jenkins,,${ }^{1,2}$ Jack S. Acton, ${ }^{3}$ Joshua Briegal, ${ }^{4}$ Daniel Bayliss ${ }^{\oplus},{ }^{5}$ François Bouchy, ${ }^{6}$ Claudia Belardi,${ }^{3}$ Edward M. Bryant,${ }^{5,7}$ Matthew R. Burleigh, ${ }^{3}$ Juan Cabrera, ${ }^{8}$ Sarah L. Casewell, ${ }^{3}$ Alexander Chaushev, ${ }^{9}$ Benjamin F. Cooke, ${ }^{5,7}$ Szilárd Csizmadia ${ }^{\odot},{ }^{8}$ Philipp Eigmüller, ${ }^{8}$ Anders Erikson, ${ }^{8}$ Emma Foxell, ${ }^{5}$ Samuel Gill, ${ }^{5,7}$ Edward Gillen ${ }^{\odot},{ }^{4} \dagger$ Michael R. Goad, ${ }^{3}$ James A. G. Jackman ${ }^{\bullet}, 5,7$ George W. King, ${ }^{5,7}$ Tom Louden ${ }^{\oplus},{ }^{5,7}$ James McCormac, ${ }^{5,7}$ Maximiliano Moyano, ${ }^{10}$ Louise D. Nielsen ${ }^{\odot}, 6$ Don Pollacco, ${ }^{5,7}$ Didier Queloz, ${ }^{4}$ Heike Rauer, ${ }^{8,9,11}$ Liam Raynard ${ }^{\oplus}{ }^{3}$ Alexis M. S. Smith, ${ }^{8}$ Maritza G. Soto, ${ }^{12}$ Rosanna H. Tilbrook, ${ }^{3}$ Ruth Titz-Weider, ${ }^{8}$ Oliver Turner ${ }^{\oplus}{ }^{6}$ Stéphane Udry, ${ }^{6}$ Simon. R. Walker, ${ }^{5}$ Christopher A. Watson, ${ }^{13}$ Richard G. West ${ }^{\oplus 5,7}$ and Peter J. Wheatley ${ }^{\oplus 5,7}$

${ }^{1}$ Departamento de Astronomía, Universidad de Chile, Casilla 36-D, 7591245, Santiago, Chile

${ }^{2}$ Centro de Astrofísica y Tecnologías Afines (CATA), Casilla 36-D, 7591245, Santiago, Chile

${ }^{3}$ Department of Physics and Astronomy, University of Leicester, University Road, Leicester LE1 7RH, UK

${ }^{4}$ Astrophysics Group, Cavendish Laboratory, J.J. Thomson Avenue, Cambridge CB3 OHE, UK

${ }^{5}$ Department of Physics, University of Warwick, Gibbet Hill Road, Coventry CV4 7AL, UK

${ }^{6}$ Observatoire de Genève, Université de Genève, 51 Ch. des Maillettes, CH-1290 Sauverny, Switzerland

${ }^{7}$ Centre for Exoplanets and Habitability, University of Warwick, Gibbet Hill Road, Coventry CV4 7AL, UK

${ }^{8}$ Institute of Planetary Research, German Aerospace Center, Rutherfordstrasse 2, D-12489 Berlin, Germany

${ }^{9}$ Center for Astronomy and Astrophysics, TU Berlin, Hardenbergstr. 36, D-10623 Berlin, Germany

${ }^{10}$ Instituto de Astronomía, Universidad Católica del Norte, Angamos 0610, 1270709 Antofagasta, Chile

${ }^{11}$ Institute of Geological Sciences, FU Berlin, Malteserstr. 74-100, D-12249 Berlin, Germany

${ }^{12}$ School of Physics and Astronomy, Queen Mary University, 327 Mile End Road, London E1 4NS, UK

${ }^{13}$ Astrophysics Research Centre, School of Mathematics and Physics, Queen's University Belfast, Belfast BT7 INN, UK

Accepted 2019 July 24. Received 2019 June 23; in original form 2019 April 13

\begin{abstract}
We report the discovery of a new ultrashort period hot Jupiter from the Next Generation Transit Survey. NGTS-6b orbits its star with a period of $21.17 \mathrm{~h}$, and has a mass and radius of $1.330_{-0.028}^{+0.024} M_{\mathrm{J}}$ and $1.271_{-0.188}^{+0.197} R_{\mathrm{J}}$, respectively, returning a planetary bulk density of $0.711_{-0.136}^{+0.214} \mathrm{~g} \mathrm{~cm}^{-3}$. Conforming to the currently known small population of ultrashort period hot Jupiters, the planet appears to orbit a metal-rich star $([\mathrm{Fe} / \mathrm{H}]=+0.11 \pm 0.09 \mathrm{dex})$. Photoevaporation models suggest the planet should have lost 5 percent of its gaseous atmosphere over the course of the $9.6 \mathrm{Gyr}$ of evolution of the system. NGTS-6b adds to the small, but growing list of ultrashort period gas giant planets, and will help us to understand the dominant formation and evolutionary mechanisms that govern this population.
\end{abstract}

Key words: planets and satellites: detection-planetary systems.

\section{INTRODUCTION}

Over the last few years, ultrashort period (USP) planets have emerged as an important subpopulation of planets, characterized solely by their proximity to the host $\operatorname{star}\left(P_{\text {orb }}<1 \mathrm{~d}\right)$. The majority

\footnotetext{
${ }^{\star}$ E-mail: jose.vines.1@gmail.com
}

$\dagger$ Winton Fellow. of the population have been detected by space-based instruments, particularly CoRoT (CoRot Team 2016) and Kepler (Borucki et al. 2010), due to the tendency of the population to heavily favour small physical sizes and masses, and therefore large densities (Charpinet et al. 2011; Pepe et al. 2013; Guenther et al. 2017; Crida et al. 2018; Santerne et al. 2018; Espinoza et al. 2019).

Ground-based radial velocity programs have found it difficult to detect these systems, since large-scale and high-cadence data sets are scarce, whereas the operational mechanics of photometric 
surveys allow for the detection of these systems. Also, the formation mechanism seems to favour small planets, and therefore both the radial velocity and transit signals are also small, making them harder to detect. However, on the plus side, other biases work in favour of these methods, since the radial velocity amplitude for a given star-planet systems increases with decreasing orbiting period, and the probability of transits rises, as well as the frequency of transits. In fact, although small USP super-Earths $\left(R_{\mathrm{p}} \leq 2 R_{\oplus}\right)$ are more common than larger planets by a factor of 5 (Winn, Sanchis-Ojeda \& Rappaport 2018), a number have been detected from ground-based photometric surveys and confirmed by radial velocity measurements, with the majority of this small sample being hot Jupiters (HJs; Southworth et al. 2009, 2015; Penev et al. 2016; Oberst et al. 2017). The population in between these two extremes of hot super-Earths and HJs have remained fairly elusive.

Models of the population of USP planets employ either photoevaporation or Roche lobe overflow of a migrating more massive planet, which strips the planet of its gaseous envelope (Valsecchi, Rasio \& Steffen 2014; Jackson et al. 2016). The migration occurs either as disc migration (Mandell, Raymond \& Sigurdsson 2007; Terquem 2014) or by dynamical interactions (Fabrycky \& Tremaine 2007). In situ formation has also been invoked to describe the population (Chiang \& Laughlin 2013). The first USP HJ, WASP19b (Hebb et al. 2010), has led to a deeper understanding of these models since a lack of explanation of why it has not lost the majority of its gaseous envelope provides strong constraints on the history of its dynamical evolution (Essick \& Weinberg 2015). Subsequent and forthcoming discoveries of USP HJ planets are providing critical constraints on the formation and evolution of close-in planets.

The Next Generation Transit Survey (NGTS; Chazelas et al. 2012; Wheatley et al. 2013; McCormac et al. 2017; Wheatley et al. 2018) has now been fully operational for over $2 \mathrm{yr}$, announcing the discovery of five new planets (Bayliss et al. 2018; Günther et al. 2018; Raynard et al. 2018; Eigmüller et al. 2019; West et al. 2019), which include a dense sub-Neptune (NGTS-4b), a sub-Jovian planet (NGTS-5b), a giant planet transiting an M dwarf star (NGTS-1b), and some new HJs (NGTS-2b, NGTS-3Ab). The dense sampling of NGTS fields over long observing seasons, combined with the high precision of individual images $(\sim 0.001$ magnitudes for a $1 \mathrm{~h}$ baseline on stars brighter than $I=14$ during dark time or $I=13$ in full moon nights), allows the detection of not only smaller transiting planets, but also those with very short periods. In this work, we report the discovery of a new USP HJ, NGTS-6b, orbiting the star NGTS-6. The paper is organized as follows; in Section 2 we describe the NGTS observations that led to the discovery, with follow-up photometry from SAAO discussed in Section 2.3 and the follow-up spectroscopy from FEROS and CORALIE discussed in Section 2.4. We analyse the nature of the star in Section 3 and discuss the modelling in Section 4. Our conclusions are highlighted in Section 5.

\section{OBSERVATIONS}

\subsection{NGTS photometry}

The NGTS is a ground-based wide-field transit survey located at the ESO Paranal Observatory in Chile, monitoring stars with $I<$ 16 (Wheatley et al. 2018). It obtains full-frame images from 12 independent telescopes, each with a field of view of $8 \mathrm{deg}^{2}$, at $13 \mathrm{~s}$ cadence. The telescopes have apertures of $20 \mathrm{~cm}$ and observe at
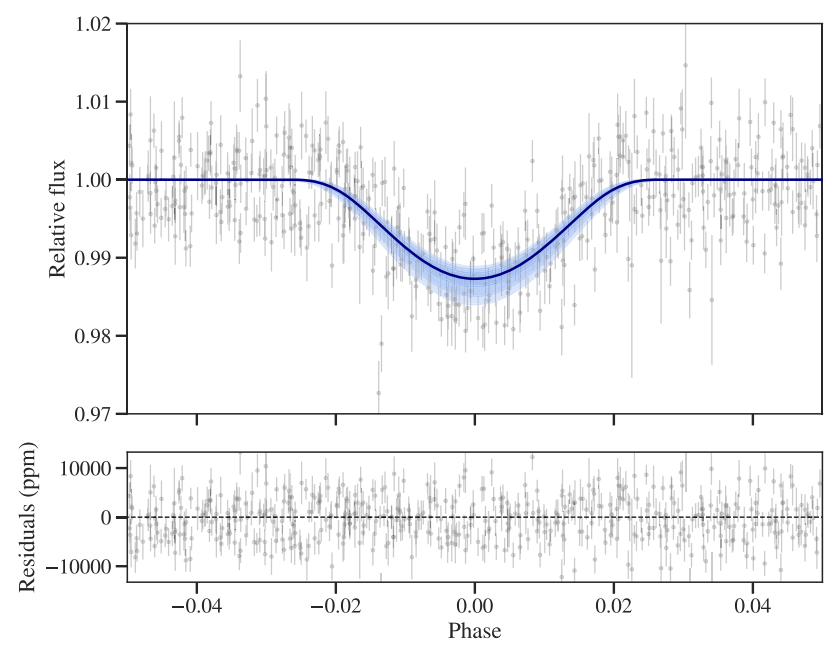

Figure 1. Light curve of NGTS photometry for NGTS-6 phase folded to the planets orbital period. The grey circles show the photometry observations binned to $10 \mathrm{~min}$ cadence and the figure is zoomed to highlight the transit. The solid blue line and blue shaded regions represent the median, $1 \sigma, 2 \sigma$, $3 \sigma$ confidence levels, respectively, of the best posterior model. Bottom: The residuals of the fit in ppm.

a bandpass of $520-890 \mathrm{~nm}$. They have a total instantaneous field of view of $96 \mathrm{deg}^{2}$. A total of $21354910 \mathrm{~s}$ exposures of the field containing NGTS- 6 were taken between the nights of 2017 August 16 and the 2018 March 23.

The NGTS data were processed using the NGTS pipeline (Wheatley et al. 2018). Aperture photometry based on the CASUTOOLS (Irwin et al. 2004) software package was used to generate the light curve using an aperture of 3 pixels, with 5 arcsec per pixel. To remove the most dominant systematic effects the SysRem algorithm (Tamuz, Mazeh \& Zucker 2005) has been utilized. Finally, using ORION, our own implementation of the BLS algorithm (Kovács, Zucker \& Mazeh 2002), a transit signal with a period of $0.882055 \mathrm{~d}$ for the star NGTS-6b was discovered. We show the transit in the NGTS phase folded light curve with the corresponding model and confidence region (see Section 4.2 for details) in Fig. 1.

\subsection{TESS photometry}

The Transiting Exoplanet Survey Satellite (TESS) is a NASAsponsored Astrophysics Explorer-class mission that is performing a wide-field survey to search for planets transiting bright stars (Ricker et al. 2015). It has four $24^{\circ} \times 24^{\circ}$ field of view cameras with four $2 \mathrm{k} \times 2 \mathrm{k}$ CCDs each, with a pixel scale of 21 arcsec per pixel and a bandpass of 600-1000 nm. Using the TESSCut tool ${ }^{1}$ we checked for available data in the TESS full frame images. NTGS-6b was observed by TESS in Sector 5 using CCD 2 of Camera 2. Between 2018 November 15 th and December 11th 1196 images with a typical cadence of $30 \mathrm{~min}$ were obtained. Using a small $2 \times 2$ pixel aperture to minimize contamination, we performed aperture photometry on the target. Long-term trends visible in the data were removed using a moving median filter. The single transit events are directly visible in the TESS data, although with the long cadence and short transit duration, not well sampled. In Fig. 2 the phase folded TESS light curve is shown.

\footnotetext{
${ }^{1}$ https://mast.stsci.edu/tesscut
} 

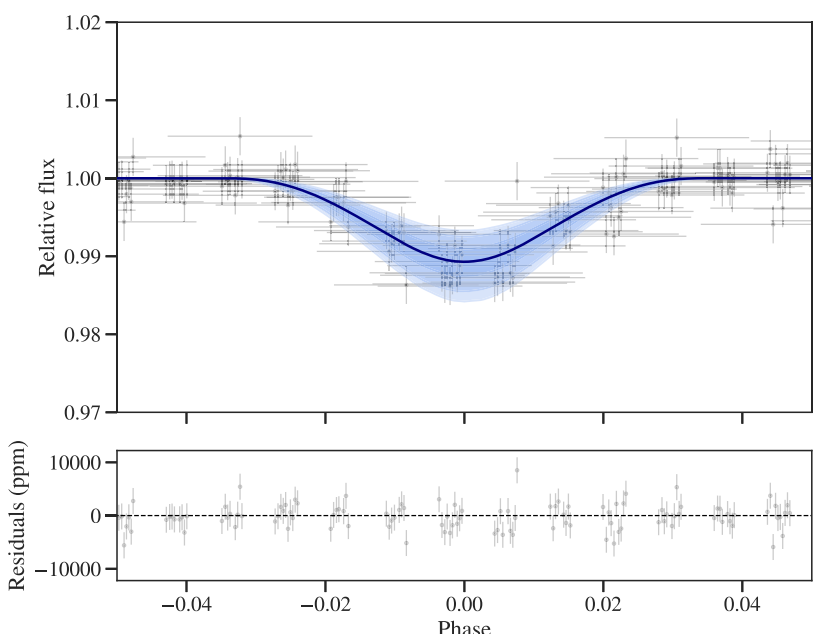

Figure 2. Phase folded TESS photometry. Horizontal errorbars show the 30 min cadence of the observations. The solid blue line is the best fit for the photometry and the blue shaded regions represent the $1 \sigma, 2 \sigma, 3 \sigma$ confidence levels. Bottom: The residuals of the fit in ppm.
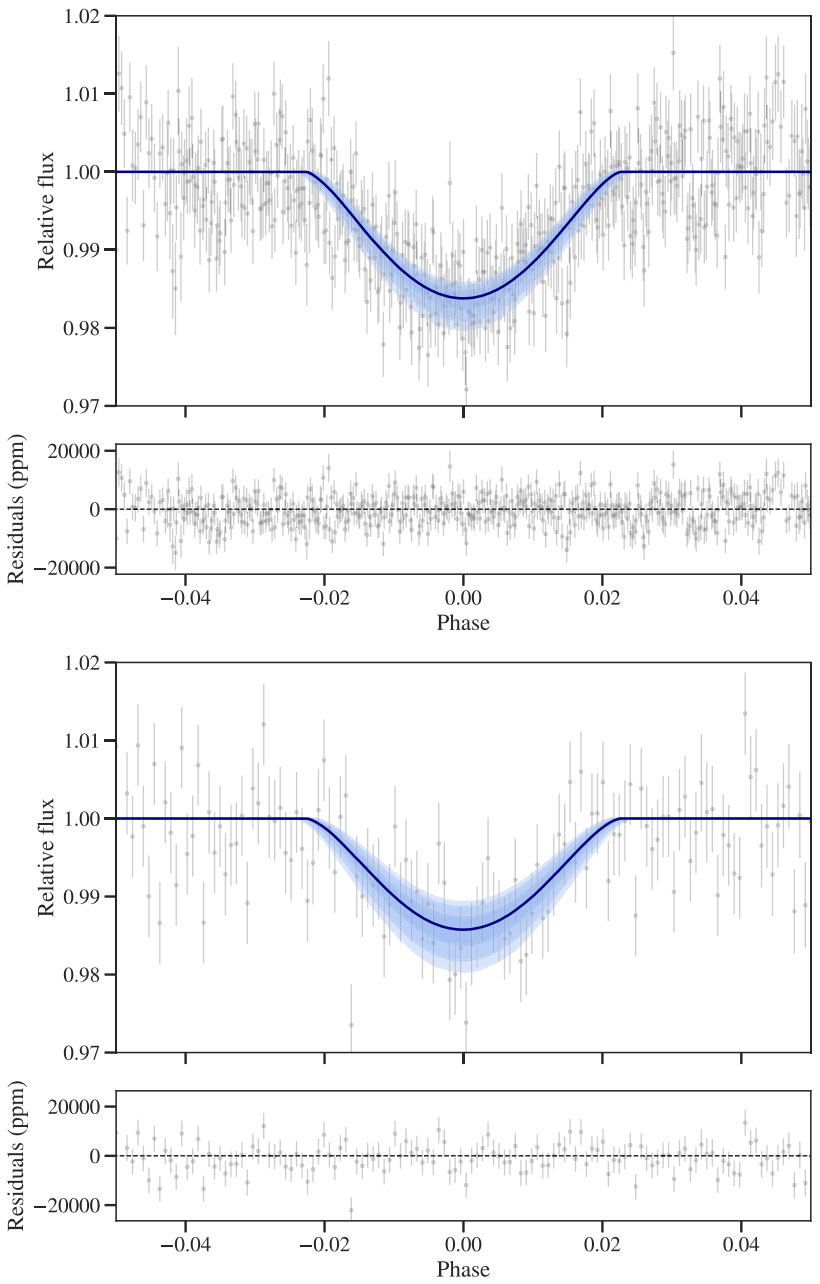

Figure 3. Top: Phase folded SAAO photometry in the $I$ and $V$ band, respectively, for (a) and (b). The solid blue line is the best fit for the photometry and the blue shaded regions represent the $1 \sigma, 2 \sigma, 3 \sigma$ confidence levels. Bottom: The residuals of the fit in ppm.
Table 1. Photometry of NGTS, SAAO I, V, and TESS for NGTS-6. The full table is available in a machine-readable format from the online journal. A portion is shown here for guidance.

\begin{tabular}{lccc}
\hline $\begin{array}{l}\text { BJD } \\
(-2450000)\end{array}$ & Relative flux & $\begin{array}{c}\text { Relative flux } \\
\text { error }\end{array}$ & INST \\
\hline$\ldots$ & $\ldots$ & $\ldots$ & $\ldots$ \\
8200.55123 & 1.0222 & 0.0169 & NGTS \\
8200.55138 & 0.9660 & 0.0168 & NGTS \\
8200.55153 & 0.9859 & 0.0169 & NGTS \\
8200.55168 & 1.0206 & 0.0171 & NGTS \\
8200.55183 & 0.9861 & 0.0169 & NGTS \\
8399.47400 & 1.0049 & 0.0044 & SAAOV \\
8399.47469 & 1.0077 & 0.0044 & SAAOV \\
8399.47539 & 0.9974 & 0.0044 & SAAOV \\
8399.47608 & 0.9982 & 0.0044 & SAAOV \\
8399.47678 & 1.0014 & 0.0043 & SAAOV \\
$\ldots$ & $\ldots$ & $\ldots$ & $\ldots$ \\
\hline
\end{tabular}

\subsection{SAAO photometric follow-up}

Three transit light curves were obtained with the 1.0-m Elizabeth telescope at the South African Astronomical Observatory (SAAO) and one of the SHOC frame-transfer CCD cameras, 'SHOC'n'awe' (Coppejans et al. 2013). The transits were collected on 2018 October 7 in $V$ band $(240 \times 60$ s exposures $)$, and on 2018 November 14 and 15 in $I$ band $(470 \times 30 \mathrm{~s}$ and $340 \times 30$ s exposures, respectively. The scale of each pixel is 0.167 arcsec). The data were reduced with the local SAAO SHOC pipeline, which is driven by PYTHON scripts running IRAF tasks (PYFITS and PYRAF), and incorporating the usual bias and flat-field calibrations. Aperture photometry was performed using the Starlink package AUTOPHOTOM.

Differential photometry was performed on each light curve using two reference stars and altering the size of the aperture to reflect the sky conditions (4px for the $V$-band light curve, $5 \mathrm{px}$ for the I-band light curve on 2018 November 14 and 3px for the light curve obtained on the following night when the conditions were considerably better).

We show phase folded light curves of the three SAAO transit events in Fig. 3 with their respective models and confidence regions. The complete light curve for all instruments is shown in Table 1

\subsection{Spectroscopic follow-up}

We obtained multi-epoch spectroscopy for NGTS-6 with two different fiber-fed high-precision Echelle spectrographs: CORALIE and FEROS. Both are located at the ESO La Silla Observatory in Chile. CORALIE is mounted on the 1.2-m Leonard Euler telescope and has a spectral resolution of $R=60000$ (Queloz et al. 2001b). FEROS is mounted on the 2.2-m MPG/ESO telescope with a spectral resolution of $R=48000$ (Kaufer et al. 1999). The spectral observations were taken between 2018 October 23 and 2019 January 8 for CORALIE and 2018 December 23 and 2019 January 2 for FEROS.

The CORALIE data were reduced using the standard data reduction pipeline (Queloz et al. 2001b) and the radial velocities were calculated by cross-correlation with a binary G2 mask. The first 20 orders of the spectrum were discarded in the cross-correlation analysis as they contain little signal. All the CORALIE spectra were also stacked to make a high-signal-to-noise spectrum for spectral analysis as presented in Section 3

FEROS data were reduced with the CERES pipeline (Brahm, Jordán \& Espinoza 2017). CERES also calculates the cross- 
Table 2. CORALIE and FEROS radial velocities for NGTS-6.

\begin{tabular}{lcccc}
\hline $\begin{array}{l}\text { BJD } \\
(-2450000)\end{array}$ & $\begin{array}{c}\mathrm{RV} \\
\left(\mathrm{km} \mathrm{s}^{-1}\right)\end{array}$ & $\begin{array}{c}\sigma_{\mathrm{RV}} \\
\left(\mathrm{km} \mathrm{s}^{-1}\right)\end{array}$ & $\begin{array}{c}\text { BIS } \\
\left(\mathrm{km} \mathrm{s}^{-1}\right)\end{array}$ & INST \\
\hline 8415.71 & -19.526 & 0.165 & -0.420 & CORALIE \\
8418.74 & -18.633 & 0.104 & -0.109 & CORALIE \\
8454.65 & -19.324 & 0.125 & 0.059 & CORALIE \\
8472.59 & -18.856 & 0.109 & 0.154 & CORALIE \\
8472.75 & -19.064 & 0.124 & 0.125 & CORALIE \\
8475.69 & -19.333 & 0.102 & - & CORALIE \\
8475.79 & -19.264 & 0.110 & - & CORALIE \\
8481.71 & -19.396 & 0.089 & -0.411 & CORALIE \\
8481.81 & -19.494 & 0.102 & 0.335 & CORALIE \\
8492.69 & -19.019 & 0.086 & -0.181 & CORALIE \\
8492.73 & -19.105 & 0.098 & -0.061 & CORALIE \\
8481.85 & -19.536 & 0.047 & 0.929 & FEROS \\
8480.75 & -19.196 & 0.026 & 0.064 & FEROS \\
8480.73 & -19.033 & 0.022 & -0.026 & FEROS \\
8484.71 & -19.189 & 0.016 & 0.050 & FEROS \\
8478.79 & -18.746 & 0.028 & 0.517 & FEROS \\
8478.60 & -19.052 & 0.025 & 0.068 & FEROS \\
8478.81 & -18.946 & 0.043 & 0.882 & FEROS \\
8478.62 & -18.874 & 0.026 & -0.054 & FEROS \\
8483.84 & -19.103 & 0.026 & -0.115 & FEROS \\
8483.67 & -19.434 & 0.017 & 0.002 & FEROS \\
\hline
\end{tabular}

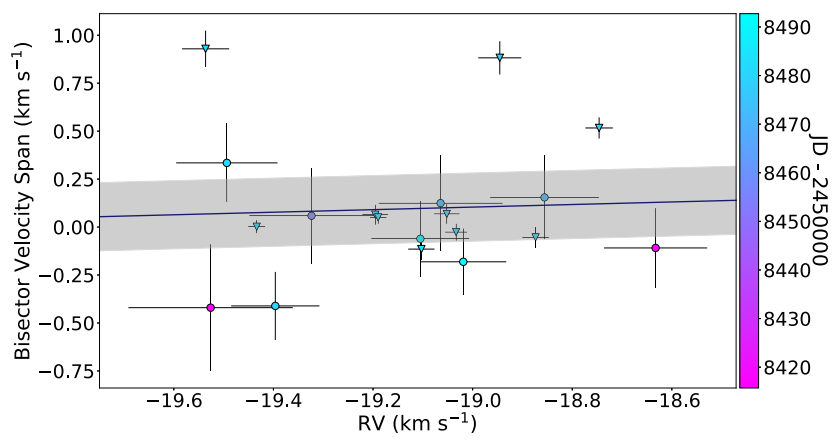

Figure 4. Bisector velocity span over radial velocity measurements colour coded by observation time. Circles are CORALIE and upside down triangles are FEROS data points. The blue solid line is a linear fit and the shaded region show the $2 \sigma$ confidence region. No correlation is detected.

correlation function $(\mathrm{CCF})$ using the reduced FEROS spectra and a binary G2 mask for each epoch and afterwards, depending on moonlight contamination, a single or double Gaussian is then fitted to find the radial velocity (depending on moonlight contamination). In the cases where the single or double Gaussian fits were unsatisfactory, a fourth-order spline was fitted to find the radial velocity instead. The radial velocities from CORALIE and FEROS are shown in Table 2 along with the uncertainties and bisector velocity span. We present a total of 21 radial velocity data points, which constrain the orbit of the planet, 11 of which were taken with CORALIE and 10 with FEROS.

As a first check we searched for a correlation between the radial velocity data and the bisector velocity span, which was calculated using the CCFs that were constructed to measure the velocity in the first place (see for example Boisse et al. 2009). Any correlation between radial velocity measurements and the bisector velocity span would cast doubts on the validity of the planet interpretation of the radial velocity variation (Queloz et al. 2001a). The bisector and radial velocities are shown in Fig. 4 along with a linear fit and a
Table 3. Comparison of empirical SpecMatch and SED fitting outputs.

\begin{tabular}{lcc}
\hline Parameter & SpecMatch & SED \\
\hline$T_{\text {eff }}(\mathrm{K})$ & $4409 \pm 70$ & $4730_{-40}^{+44}$ \\
$\log g$ & $4.63 \pm 0.12$ & $4.7_{-0.7}^{+1.1}$ \\
$R_{\mathrm{S}}\left(\mathrm{R}_{\odot}\right)$ & $0.72 \pm 0.1$ & $0.754 \pm 0.013$ \\
{$[\mathrm{Fe} / \mathrm{H}]$} & $0.11 \pm 0.09$ & - \\
$(\dagger) M_{\mathrm{S}}\left(\mathrm{M}_{\odot}\right)$ & $0.72 \pm 0.08$ & $0.767 \pm 0.025$ \\
$(\dagger)$ Age $(\mathrm{Gyr})$ & $9.61 \pm 0.17$ & $9.77_{-0.54}^{+0.25}$ \\
Distance $(\mathrm{pc})$ & - & $308 \pm 2$ \\
$A_{\mathrm{V}}$ & - & $0.017 \pm 0.010$ \\
\hline
\end{tabular}

Note. In the case of SED fitting, the parameters with $(\dagger)$ were calculated using the isochrones package.

$2 \sigma$ confidence region, with no correlation detected. We calculated the Pearson $r$ coefficient to be 0.05 , which reaffirms our claim of no significant linear correlation being present.

\section{STELLAR PARAMETERS}

Given the host star is relatively faint ( $V=14.087$ ), the highresolution Echelle spectra used for the calculation of the radial velocity measurements have SNR too low for the accurate measurement of the equivalent of individual absorption lines, therefore it is not possible to obtain a constrained solution from the SPECIES code (Soto \& Jenkins 2018) for the stellar bulk parameters. Therefore, we used two methods: the empirical SpecMatch tool (Yee, Petigura \& von Braun 2017) with the combined CORALIE spectra, which given the observing conditions have a higher SNR than the FEROS spectra, and an Spectral Energy Distribution (SED) fit of the star detailed in Section 3.1. The output of the two employed methods are shown in Table 3

We finally adopt the results from the SED fitting routine for $T_{\text {eff }}, \log g$, and $R_{\mathrm{s}}$, and the metallicity from the SpecMatch tool. We used these parameters to calculate the mass and age of the star, using the isochrones package (Morton 2015). The projected rotational velocity, vsini, was estimated using the SPECIES code. We combined the individual spectra obtained with Coralie to obtain a high-S/N spectrum from the target, and created synthetic absorption line profiles for four iron lines in the spectrum, using the ATLAS9 model atmospheres (Castelli \& Kurucz 2004) and the atmospheric parameters previously obtained. We then broaden the absorption lines, by adjusting the rotational velocity, until they matched the observations. More details about this procedure can be found in Soto \& Jenkins (2018). The obtained vsini is listed in Table 4. Thus we conclude that NGTS-6 is an old K dwarf with an effective temperature of $4730_{-40}^{+44} \mathrm{~K}, \log g$ of $4.7_{-0.7}^{+1.1}$ dex, $[\mathrm{Fe} / \mathrm{H}]$ of $0.11 \pm 0.09 \mathrm{dex}$, radius of $0.754 \pm 0.013 \mathrm{R}_{\odot}$, mass of $0.767 \pm 0.025 \mathrm{M}_{\odot}$, and age of $9.77_{-0.54}^{+0.25}$ Gyr. We show NGTS-6 catalogue information and stellar parameters in Table 4.

\subsection{SED fitting and dilution}

Using Gaia DR2 we identified a neighbouring source 5.4 arcsec away that could be contaminating our photometry. Therefore, in order to determine the level of dilution from this source we performed SED fitting of both stars using the PHOENIX v2 models (Husser et al. 2013). This was done following a method similar to Gillen et al. (2017), by first generating a grid of bandpass fluxes in $T_{\text {eff }}$ and $\log g$ space. To overcome the issue of possible blending in catalogue photometry (due to the small separation of the two sources) we fit only to the Pan-STARRS, 2MASS, Gaia, 
Table 4. Stellar properties for NGTS-6.

\begin{tabular}{|c|c|c|}
\hline Property & Value & Source \\
\hline \multicolumn{3}{|c|}{ Astrometric properties } \\
\hline RA & $05^{\mathrm{h}} 03^{\mathrm{m}} 10^{\mathrm{s}} .90$ & 2MASS \\
\hline Dec. & $-30^{\circ} 23^{\prime} 57^{\prime \prime} 6420$ & 2MASS \\
\hline 2MASS I.D. & 05031090-3023576 & 2MASS \\
\hline Gaia DR2 I.D. & 4875693023844840448 & Gaia \\
\hline TIC ID & 1528696 & TESS \\
\hline$\mu_{\mathrm{RA}}\left(\operatorname{mas~}^{-1}\right)$ & $-6.0 \pm 7.0$ & UCAC4 \\
\hline$\mu_{\text {Dec. }}\left(\right.$ mas y $\left.^{-1}\right)$ & $-33.5 \pm 10.1$ & UCAC4 \\
\hline$\varpi$ (mas) & $3.215 \pm 0.015$ & Gaia \\
\hline \multicolumn{3}{|c|}{ Photometric properties } \\
\hline$V(\mathrm{mag})$ & $14.087 \pm 0.021$ & APASS \\
\hline$B$ (mag) & $15.171 \pm 0.014$ & APASS \\
\hline$g(\mathrm{mag})$ & $14.639 \pm 0.058$ & APASS \\
\hline$r$ (mag) & $13.703 \pm 0.032$ & APASS \\
\hline$i(\mathrm{mag})$ & $13.378 \pm 0.057$ & APASS \\
\hline$r_{\mathrm{P} 1}(\mathrm{mag})$ & $13.751 \pm 0.002$ & Pan-STARRS \\
\hline$z_{\mathrm{P} 1}(\mathrm{mag})$ & $13.364 \pm 0.002$ & Pan-STARRS \\
\hline$y_{\mathrm{P} 1}(\mathrm{mag})$ & $13.250 \pm 0.006$ & Pan-STARRS \\
\hline$G$ (mag) & $13.818 \pm 0.001$ & Gaia \\
\hline $\mathrm{BP}$ (mag) & $14.401 \pm 0.003$ & Gaia \\
\hline RP (mag) & $13.113 \pm 0.002$ & Gaia \\
\hline NGTS (mag) & 13.460 & This work \\
\hline TESS (mag) & 13.070 & TESS \\
\hline$J(\mathrm{mag})$ & $12.222 \pm 0.033$ & 2MASS \\
\hline$H$ (mag) & $11.767 \pm 0.038$ & 2MASS \\
\hline$K$ (mag) & $11.650 \pm 0.032$ & 2MASS \\
\hline W1 (mag) & $11.609 \pm 0.028$ & WISE \\
\hline W2 (mag) & $11.688 \pm 0.029$ & WISE \\
\hline \multicolumn{3}{|l|}{ Derived properties } \\
\hline$T_{\text {eff }}(\mathrm{K})$ & $4730_{-40}^{+44}$ & SED fitting \\
\hline $\log g$ & $4.7_{-0.7}^{+1.1}$ & SED fitting \\
\hline$[\mathrm{Fe} / \mathrm{H}]$ & $0.11 \pm 0.09$ & CORALIE spectra \\
\hline $\operatorname{vsini}\left(\mathrm{km} \mathrm{s}^{-1}\right)$ & $2.851 \pm 0.431$ & CORALIE spectra \\
\hline$\gamma_{\text {CORALIE }}\left(\mathrm{km} \mathrm{s}^{-1}\right)$ & $-19.137_{-0.018}^{+0.018}$ & Global Modelling \\
\hline$\gamma_{\text {FEROS }}\left(\mathrm{km} \mathrm{s}^{-1}\right)$ & $-19.142 \pm 0.010$ & Global Modelling \\
\hline$\sigma_{\text {CORALIE }}\left(\mathrm{km} \mathrm{s}^{-1}\right)$ & $0.000_{-0.041}^{+0.039}$ & Global Modelling \\
\hline$\sigma_{\text {FEROS }}\left(\mathrm{km} \mathrm{s}^{-1}\right)$ & $0.036_{-0.094}^{+0.023}$ & Global Modelling \\
\hline$L_{\mathrm{S}}\left(\mathrm{L}_{\odot}\right)$ & $0.256 \pm 0.009$ & SED fitting \\
\hline$M_{\mathrm{S}}\left(\mathrm{M}_{\odot}\right)$ & $0.767 \pm 0.025$ & SED fitting \\
\hline$R_{\mathrm{S}}\left(\mathrm{R}_{\odot}\right)$ & $0.754 \pm 0.013$ & SED fitting \\
\hline$\rho\left(\mathrm{g} \mathrm{cm}^{-3}\right)$ & $3.9304_{-0.0812}^{+0.0815}$ & Global Modeling \\
\hline Age & $9.77_{-0.54}^{+0.25}$ & SED fitting \\
\hline Distance (pc) & $311.042 \pm 1.432$ & Gaia \\
\hline
\end{tabular}

Note. 2MASS (Skrutskie et al. 2006); UCAC4 (Zacharias et al. 2013); APASS (Henden \& Munari 2014); WISE (Wright et al. 2010); Gaia (Gaia Collaboration 2016, 2018); TESS (Stassun et al. 2018) Pan-STARRS (Tonry et al. 2012; Chambers et al. 2016).

and WISE photometry for each source. We fit for the $T_{\text {eff }}, \log g$, radius, distance, $V$-band extinction $\mathrm{A}_{V}$, and an uncertainty term $\sigma$ to account for underestimated catalogue uncertainties. We have used a Gaussian prior on the distance, constrained by the values calculated by Bailer-Jones et al. (2018) using Gaia DR2 data. We limit the $V$-band extinction of each source to a maximum value of 0.032, taken from the Galactic dust reddening maps of Schlafly \& Finkbeiner (2011). Before fitting we verified that neither source was flagged as extended in the catalogues used. Due to the larger size of the TESS aperture, an extra source 15.9 arcsec was identified as possibly contributing light. Consequently, we also performed SED fitting on this source and included it for the TESS dilution value only. To sample the posterior parameter space for each source we used
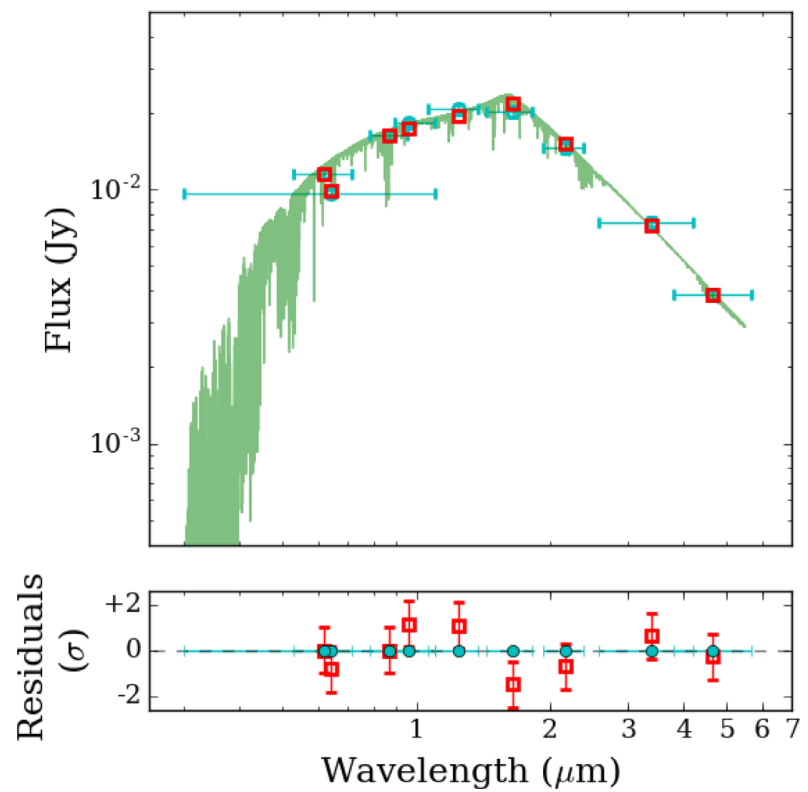

Figure 5. Top: The best-fitting PHOENIX v2 SED model, obtained from fitting to unblended Pan-STARRS, Gaia, 2MASS, and WISE photometry. The cyan and red points indicate the catalogue and synthetic photometry, respectively. The horizontal error bars indicate the spectral coverage of each band. Bottom: Residuals of the synthetic photometry, normalized to the catalogue errors.

EMCEE (Foreman-Mackey et al. 2013) to create a Markov Chain Monte Carlo (MCMC) process for our fitting. In this process we used 100 walkers for 50000 steps and discarded the first 10000 as a burn in. The best-fitting SED model for NGTS-6 is shown in Fig. 5 and the values are given in Table 4 .

To estimate the level of dilution in each bandpass we convolved the SED model for each star with the specified filter, taking the ratio of the measured synthetic fluxes as the dilution value. In order to sample the full range of dilutions and thus provide an informative prior we draw our SED models directly from the posterior distribution for each star The calculated dilutions are $D_{\mathrm{NGTS}}=0.056 \pm 0.002, D_{\mathrm{SAAOV}}=0.025 \pm 0.002, D_{\mathrm{SAAOI}}=$ $0.085 \pm 0.003$, and $D_{\text {TESS }}=0.077 \pm 0.003$. With these results we generate priors for the dilution in our light curves, which are used in the transit fitting.

\section{DATA MODELLING}

\subsection{Pure radial velocity modelling}

First, a pure radial velocity search and model fit was made using the EMPEROR algorithm (Peña Rojas \& Jenkins, in preparation). EMPEROR is a public, PYTHON-based code that is designed to search for small signals in radial velocity data using Bayesian modelling techniques and MCMC tools. The algorithm allows for correlated noise models to be incorporated into the modelling, in particular moving averages of order selected by the user. The code uses the affine-invariant EMCEE sampler in parallel tempering mode to efficiently sample highly multimodal posteriors.

In order to first test if the signal was present in the data without the use of inputs from the photometry, as a test of signal independence, we employed six chains with different temperature values $(\beta=$ $1.0,0.66,0.44,0.29,0.19$, and 0.13$)$. The chain length was set 


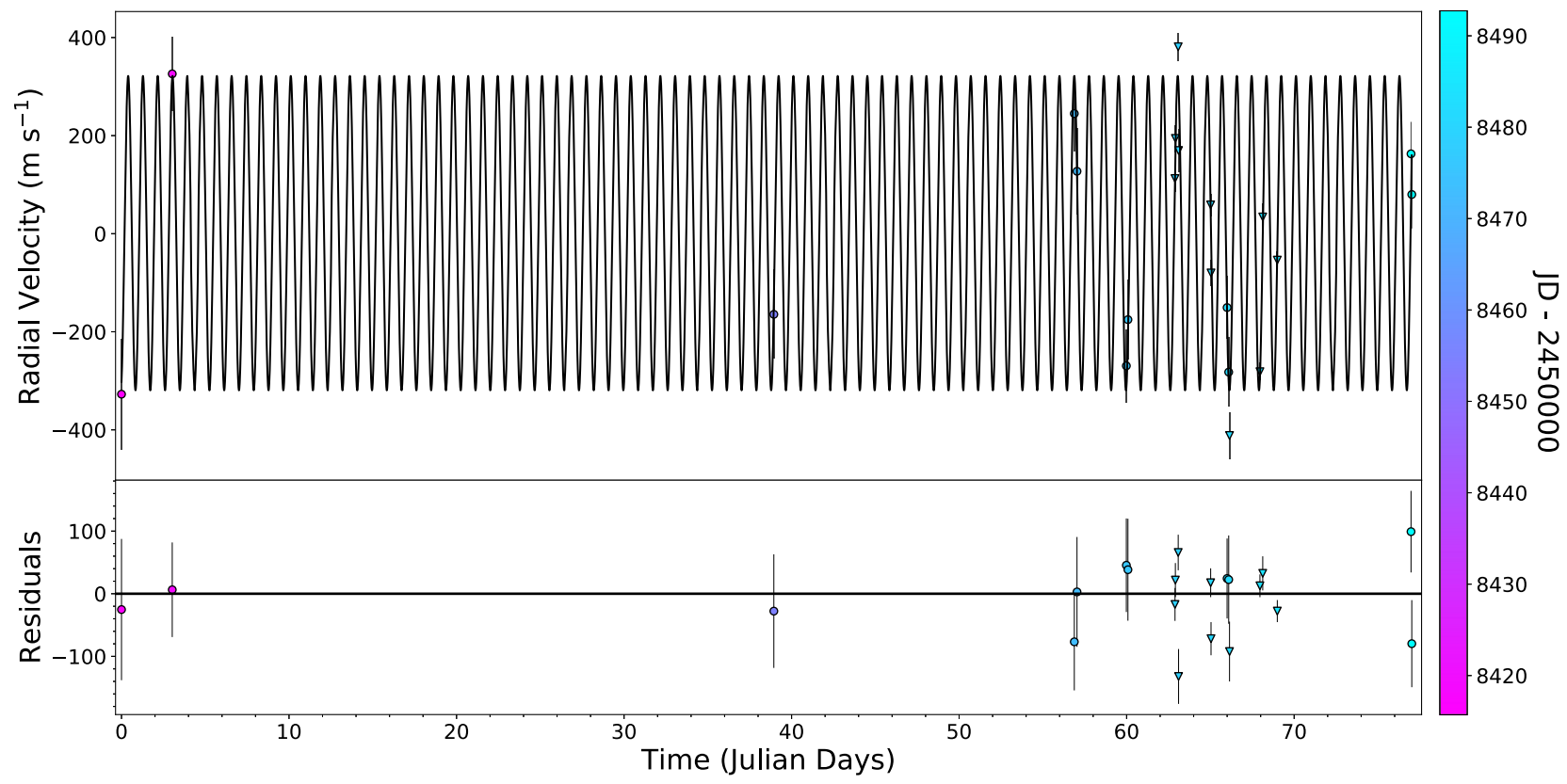

Figure 6. Top: The full timeseries of NGTS-6 radial velocity observations, colour coded by observation time. Circles are CORALIE data points and upside down triangles are FEROS. The solid black line is the best Keplerian fit. Bottom: The residuals of the fit.

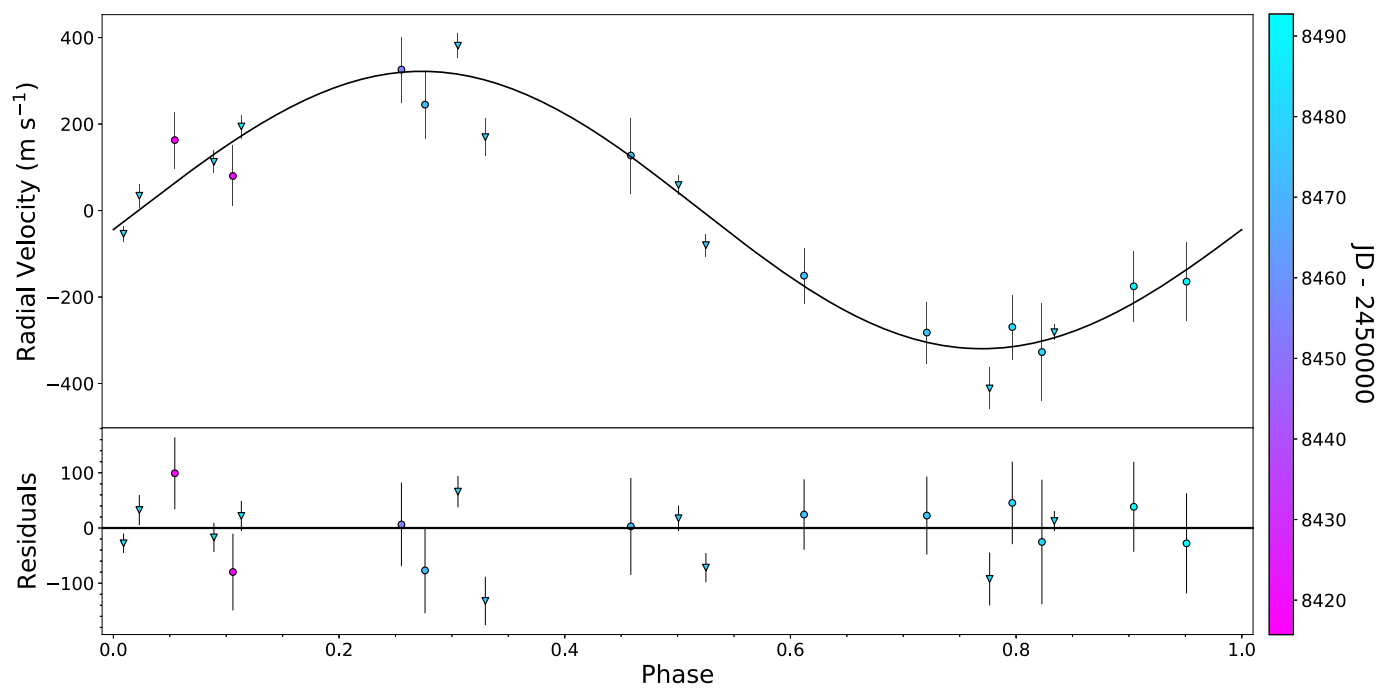

Figure 7. Top: NGTS-6 radial velocity measurements and model in orbital phase, colour coded by obervation times. Circles are CORALIE data points and upside down triangles are FEROS. The solid black line is the fit to best orbital solution. Bottom: Residuals of the fit.

to 15000 steps and each chain had 150 walkers in the ensemble, giving rise to a total chain length of 13.5 million steps. A burn-in of 7500 steps was also used. A first-order moving average correlated noise model was used to model the high-frequency noise in the velocity data set, and the priors were set to be the standard priors as explained in the EMPEROR manuscript and on the GitHub page. ${ }^{2}$ In automatic mode, EMPEROR detects the planet's orbital signature with a Bayes Factor value of 5, highly significant, in the combined FEROS+CORALIE data, confirming the existence of the planet. The best fit made by EMPEROR is shown in Fig. 6 and the phase

${ }^{2}$ https://github.com/ReddTea/astroEMPEROR folded curve in Fig. 7. No additional signal was detected. The bestfitting model from EMPEROR with respective uncertainties were used as Gaussian priors to determine a global model for this system.

\subsection{Global modelling}

For the global joint photometry and radial velocity modelling we used JULIET (Espinoza, Kossakowski \& Brahm 2018). JULIET is a PYTHON tool capable of analysis of transits, radial velocities, or both. It allows the analysis of multiple photometry and radial velocity instruments at the same time using Nested Sampling, Importance Nested Sampling, and Dynamic Nested Sampling algorithms. For the transit models, JULIET uses BATMAN (Kreidberg 2015), which 
Table 5. Planetary properties for NGTS-6b.

\begin{tabular}{lc}
\hline Property & Value \\
\hline$P(\mathrm{~d})$ & $0.882059 \pm 0.0000008$ \\
$T_{\mathrm{C}}(\mathrm{BJD}-2450000)$ & $7982.3784 \pm 0.0003$ \\
$a / R_{*}$ & $4.784_{-0.048}^{+0.043}$ \\
$b$ & $0.976_{-0.020}^{+0.015}$ \\
$K\left(\mathrm{~km} \mathrm{~s}^{-1}\right)$ & $0.322 \pm 0.008$ \\
$e$ & $0.0($ fixed $)$ \\
$M_{\mathrm{p}}\left(M_{\mathrm{J}}\right)$ & $1.339 \pm 0.028$ \\
$R_{\mathrm{p}}\left(R_{\mathrm{J}}\right)$ & $1.326_{-0.112}^{+0.097}$ \\
$\rho_{\mathrm{p}}\left(\mathrm{g} \mathrm{cm}{ }^{-3}\right)$ & $0.711_{-0.136}^{+0.214}$ \\
$a(\mathrm{au})$ & $0.01677 \pm 0.00032$ \\
inc $(\mathrm{deg})$ & $78.231_{-0.210}^{+0.262}$ \\
$T_{\mathrm{eq}}(\mathrm{K})$ & $1283.90_{-12.14}^{+12.49}$ \\
\hline
\end{tabular}

has flexible options, in particular for limb-darkening laws. The Keplerian signal model is provided by radvel (Fulton et al. 2018). Finally for our JULIET run, given the high dimensionality of the model (29 free parameters between two radial velocity and four photometry instruments) we used DYNESTY for Dynamic Nested Sampling as it has proven to be more efficient than regular Nested Sampling under these conditions.

The radial velocity fit made by EMPEROR shows a low-eccentricity orbit $(e<0.01)$ thus for the JULIET modelling we decided to fix the eccentricity to 0 . Since we have 213549 NGTS photometry data points plus SAAO photometry in the $V$ and $I$ bands and TESS photometry, fitting such a large light curve is resource intensive, so we first binned the NGTS data in 10 min cadence bins and then performed the fit with the binned data, supersampling the model light curve to 10 min exposure times with 30 points in each bin. We also employed supersampling for the $30 \mathrm{~min}$ TESS observations. For the limb darkening we assumed a quadratic law for each instrument. Using JULIET's and the SED fitting output and assuming a Jupiter's Bond albedo of 0.503 ( $\mathrm{Li}$ et al. 2018) we calculated the equilibrium temperature of NGTS- $6 \mathrm{~b}$ to be $1283.90_{-12.14}^{+12.49} \mathrm{~K}$. The parameters for the best fit are presented in Table 5 and in Fig. 8 we show a corner plot with the main planetary parameters.

The light curves showcased in Section 2.3 show a clear V shape transit, suggesting the system is in fact, grazing. This introduces a strong degeneracy between the planet-to-star radius ratio and impact parameter and can produce extreme results (such as an extremely inflated planet). In order to address this issue, a prior for the stellar density constructed from the results of the SED fitting routine was used within JULIET, which allowed us to better decorrelate those two parameters and thus get more realistic results for the parameters of the planet.

\section{DISCUSSION AND CONCLUSION}

We report the discovery of NGTS-6b, a grazing transit USP HJ with a period of $21.17 \mathrm{~h}$, mass of $1.339 \pm 0.028 M_{\mathrm{J}}$, and radius of $1.326_{-0.210}^{+0.214} R_{\mathrm{J}}$, and the first USP HJ from the NGTS. We analysed the joint photometry and radial velocity data using JULIET, testing its modelling abilities when given a likely grazing transit. There are only a handful of USP planets in the literature, of which only six are giant planets with $R_{\mathrm{p}}>8 \mathrm{R}_{\oplus}$ (WASP-18b, WASP-43b, WASP103b, HATS-18b, KELT-16b, and WASP-19b) and therefore our discovery of NGTS-6b represents a significant addition to this extreme population. In Figs 9-11 we show that NGTS-6b sits at

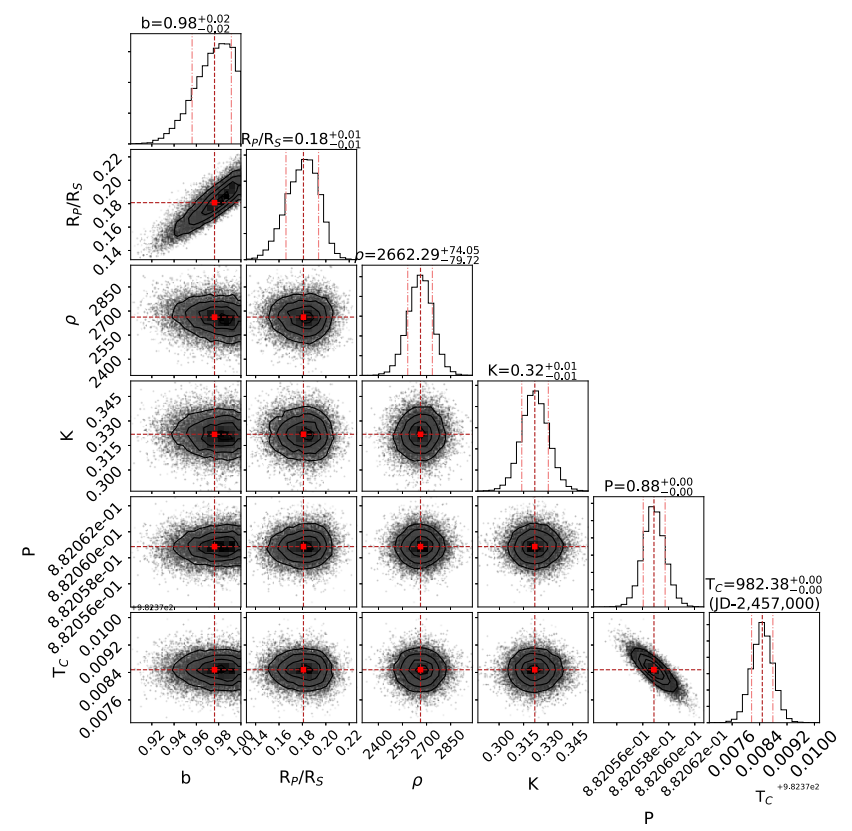

Figure 8. JULIET posterior distributions for the main planetary parameters. The red dashed lines are the median of each distribution and the dash-dotted lines represent the $1 \sigma$ confidence interval. A correlation between the impact parameter and the planet to star radius is expected due to the grazing nature of the system.

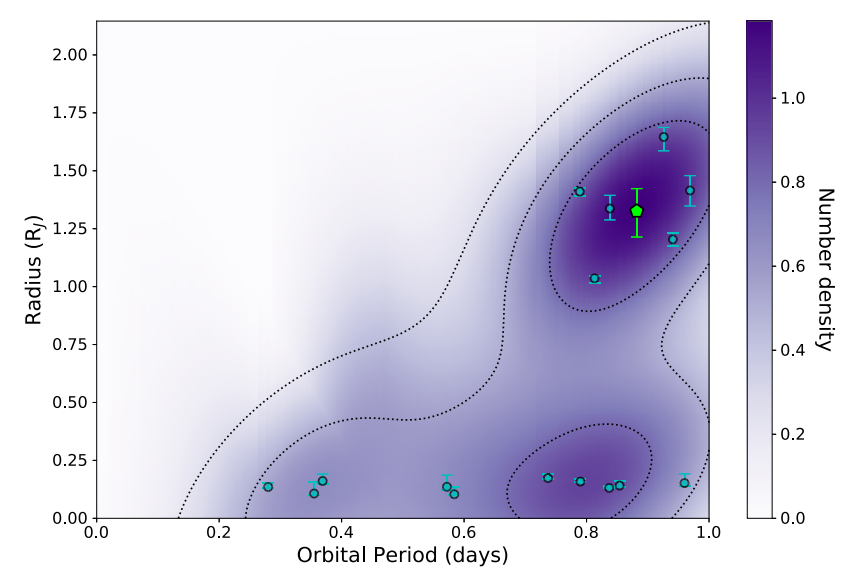

Figure 9. Planetary radius against orbital period. Plotted are all USP planets and UHJs from the well-studied transiting planets catalogue that have both measured mass and radius. The dark contours and purple shading highlight the planet number density of the sample. The green pentagon shows the position of NGTS-6b.

the centre of the distinct clump of UHJs, and thus adds weight to this being a distinct population.

We investigated photoevaporation of the planet, applying empirical relations from Jackson, Davis \& Wheatley (2012), linking the ratio of the X-ray and bolometric luminosities, $L_{X} / L_{\text {bol }}$, with stellar age. Using the isochrones-derived age of $9.77 \mathrm{Gyr}$ yields an estimate of $L_{\mathrm{X}} / L_{\mathrm{bol}}=1.0 \times 10^{-5}$ at the current epoch. This corresponds to an X-ray luminosity $L_{\mathrm{X}}=6 \times 10^{27} \mathrm{erg} \mathrm{s}^{-1}$, or a flux at Earth of $5 \times 10^{-16} \mathrm{erg} \mathrm{s}^{-1} \mathrm{~cm}^{-2}$. Such a flux would require a very deep observation with current generation X-ray telescopes in order to detect the star. Using the energy-limited method of estimating atmospheric mass-loss (Watson, Donahue \& Walker 


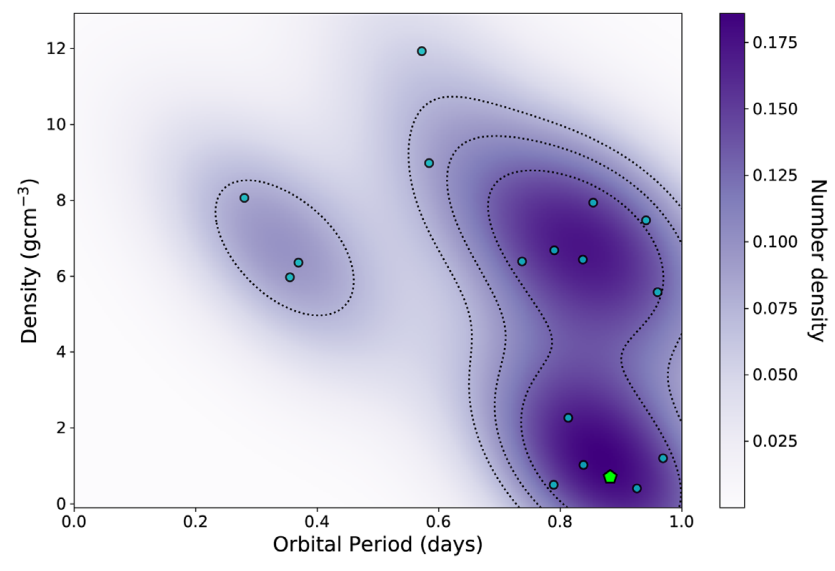

Figure 10. Similar to Fig. 9 except we show the planet bulk density against orbital period.

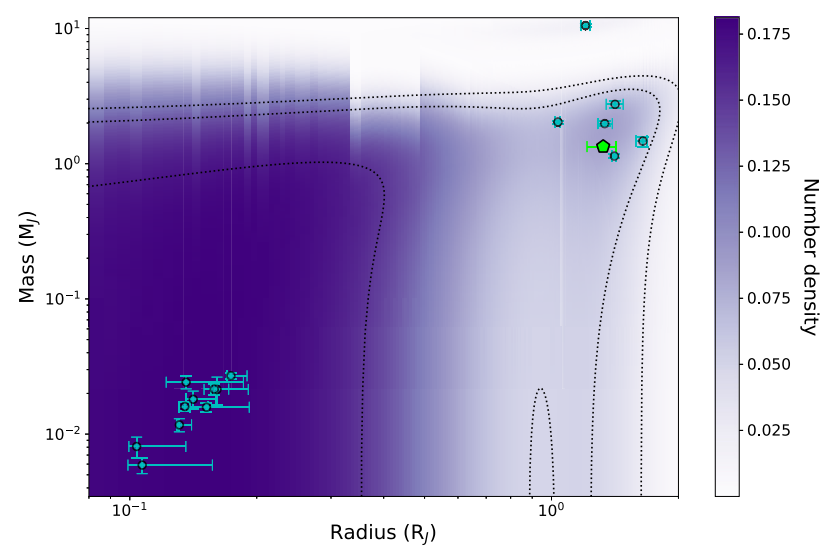

Figure 11. Similar to Figs 9 and 10 except here we show the planet mass against planet radius.

1981; Erkaev et al. 2007), our estimate of $L_{X}$ yields a mass-loss rate of $1 \times 10^{11} \mathrm{~g} \mathrm{~s}^{-1}$. By integrating the mass-loss rate across the lifetime of the star (following the X-ray evolution described by Jackson et al. 2012) we estimate a total mass-loss of about 5 per cent. This is not enough to have significantly evolved the planet, in line with theoretical studies of HJs (e.g. Murray-Clay, Chiang \& Murray 2009; Owen \& Jackson 2012).

We found the host star is likely metal-rich, with a value for the iron abundance $[\mathrm{Fe} / \mathrm{H}]$ of $+0.11 \pm 0.09$ dex. It is well established that gas giant planets favour metal-rich stars (Gonzalez 1997; Santos et al. 2002; Fischer \& Valenti 2005; Wang \& Fischer 2015), and also short period gas giants, including the $\mathrm{HJ}$ population, also appear even more metal enhanced when compared to their longer period cousins (Jenkins et al. 2017; Maldonado, Villaver \& Eiroa 2018). This trend appears to continue into the USP planet population also (Winn et al. 2017). Approximately 50 per cent of the USP HJ planets host stars are found to have supersolar metallicities $([\mathrm{Fe} / \mathrm{H}] \geq$ +0.1 dex), whereas for the smaller super-Earth population, only 30 percent orbit such stars. Since the USP HJ sample is still significantly smaller than the super-Earth sample, NGTS-6b adds statistical weight to this finding, and the conclusion that this points towards is that both these populations form through core accretion processes (Matsuo et al. 2007), with the HJ sample forming at relatively large separations from their host stars, and later migrating inwards either through disc driven migration (Mandell et al. 2007; Terquem 2014) or high-eccentricity processes like planet-planet scattering (Rasio \& Ford 1996; Ford, Havlickova \& Rasio 2001; Papaloizou \& Terquem 2001; Ford \& Rasio 2008).

In Mazeh, Holczer \& Faigler (2016) they defined the upper and lower boundaries of the so-called 'Neptune desert' region, where it was earlier found that there exists a lack of intermediate mass planets (see Szabó \& Kiss 2011; Lundkvist et al. 2016). Since photoevaporation does not appear to have affected the evolution of NGTS-6b significantly, in line with studies of other HJs that define the upper boundary of this desert (Demangeon et al. 2018), the planet may have arrived at its current location through higheccentricity evolution. Owen \& Lai (2018) suggest that a combination of tidal driven migration to short period orbits through dynamical interactions with other planetary-mass bodies in the system, coupled with photoevaporation of planetary atmospheres can readily describe this sub-Jovian boundary.

The likelihood of a planet-planet scattering evolutionary scenario for NGTS-6b may also be bolstered if the star is indeed metalrich. We can envisage that the planet core quickly grew to a size that crossed the critical core mass limit (Mizuno 1980), allowing significant accretion of the surrounding gas in the disc. Yet with a metal-rich protoplanetary disc there would be a high fraction of solids remaining for further planetesimals to form close enough to the young NGTS-6b that they could interact and be scattered to wider orbits, or ejected completely from the system (Petrovich, Deibert \& Wu 2019). Most USP planets are associated with longer period companions (Sanchis-Ojeda et al. 2014; Adams et al. 2017; Winn et al. 2018), where 52 per cent \pm 5 per cent of HJs have additional, longer period companions (Bryan et al. 2016). A concerted effort to search for additional planets further out in the system, whilst constraining better the orbit of NGTS-6b, may shed some light on these scenarios.

\section{ACKNOWLEDGEMENTS}

Based on data collected under the NGTS project at the ESO La Silla Paranal Observatory. The NGTS facility is operated by the consortium institutes with support from the UK Science and Technology Facilities Council (STFC) project ST/M001962/1. This paper includes data collected by the TESS mission. Funding for the TESS mission is provided by the NASA Explorer Program. This paper uses observations made at the South African Astronomical Observatory (SAAO). PE and AC.

JIV acknowledges support of CONICYT-PFCHA/Doctorado Nacional-21191829, Chile. JSJ acknowledges support by Fondecyt grant 1161218 and partial support by CATA-Basal (PB06, CONICYT). Contributions at the University of Geneva by DB, FB, $\mathrm{BC}, \mathrm{LM}$, and SU were carried out within the framework of the National Centre for Competence in Research 'PlanetS' supported by the Swiss National Science Foundation (SNSF). The contributions at the University of Warwick by PJW, RGW, DLP, FF, DA, BTG, and TL have been supported by STFC through consolidated grants ST/L000733/1 and ST/P000495/1. The contributions at the University of Leicester by MGW and MRB have been supported by STFC through consolidated grant ST/N000757/1. TL was also supported by STFC studentship 1226157. MNG is supported by the STFC award reference 1490409 as well as the Isaac Newton Studentship. EG gratefully acknowledges support from Winton Philanthropies in the form of a Winton Exoplanet Fellowship. SLC acknolwedges support from an STFC Ernest Rutherford Fellowship. PE, ACh, and HR acknowledge the support of the DFG 
priority program SPP 1992 'Exploring the Diversity of Extrasolar Planets' (RA 714/13-1). This project has received funding from the European Research Council (ERC) under the European Union's Horizon 2020 research and innovation programme (grant agreement No 681601). The research leading to these results has received funding from the European Research Council under the European Union's Seventh Framework Programme (FP/2007-2013) / ERC Grant Agreement n. 320964 (WDTracer). We thank Marissa Kotze (SAAO) for developing the SHOC camera data reduction pipeline We thank the Swiss National Science Foundation (SNSF) and the Geneva University for their continuous support to our planet search programs. This work has been in particular carried out in the frame of the National Centre for Competence in Research PlanetS supported by the Swiss National Science Foundation (SNSF). This publication makes use of The Data \& Analysis Center for Exoplanets (DACE), which is a facility based at the University of Geneva $(\mathrm{CH})$ dedicated to extrasolar planets data visualization, exchange and analysis. DACE is a platform of the Swiss National Centre of Competence in Research (NCCR) PlanetS, federating the Swiss expertise in Exoplanet research. The DACE platform is available at https://dace.unige.ch. The Pan-STARRS1 Surveys (PS1) and the PS1 public science archive have been made possible through contributions by the Institute for Astronomy, the University of Hawaii, the Pan-STARRS Project Office, the MaxPlanck Society and its participating institutes, the Max Planck Institute for Astronomy, Heidelberg and the Max Planck Institute for Extraterrestrial Physics, Garching, The Johns Hopkins University, Durham University, the University of Edinburgh, the Queen's University Belfast, the Harvard-Smithsonian Center for Astrophysics, the Las Cumbres Observatory Global Telescope Network Incorporated, the National Central University of Taiwan, the Space Telescope Science Institute, the National Aeronautics and Space Administration under Grant No. NNX08AR22G issued through the Planetary Science Division of the NASA Science Mission Directorate, the National Science Foundation Grant No. AST-1238877, the University of Maryland, Eotvos Lorand University (ELTE), the Los Alamos National Laboratory, and the Gordon and Betty Moore Foundation.

\section{REFERENCES}

Adams E. R. et al., 2017, AJ, 153, 82

Bailer-Jones C. A. L., Rybizki J., Fouesneau M., Mantelet G., Andrae R., 2018, AJ, 156, 58

Bayliss D. et al., 2018, MNRAS, 475, 4467

Boisse I. et al., 2009, A\&A, 495, 959

Borucki W. J. et al., 2010, Science, 327, 977

Brahm R., Jordán A., Espinoza N., 2017, PASP, 129, 034002

Bryan M. L. et al., 2016, ApJ, 821, 89

Castelli F., Kurucz R. L., 2003, IAU Symposium, 210, A20

Chambers K. C. et al., 2016, preprint (arXiv:1612.05560)

Charpinet S. et al., 2011, Nature, 480, 496

Chazelas B. et al., 2012, in Stepp L. M., Gilmozzi R., Hall H. J., eds, Proc. SPIE Conf. Ser. Vol. 8444, Ground-based and Airborne Telescopes IV. SPIE, Bellingham, p. 84440E

Chiang E., Laughlin G., 2013, MNRAS, 431, 3444

Coppejans R. et al., 2013, PASP, 125, 976

CoRot Team, 2016, The CoRoT Legacy Book, EDP Open, Les Ulis

Crida A., Ligi R., Dorn C., Borsa F., Lebreton Y., 2018, Res. Notes Am. Astron. Soc., 2, 172

Demangeon O. D. S. et al., 2018, A\&A, 610, A63

Eigmüller P. et al., 2019, A\&A, 625, A142

Erkaev N. V., Kulikov Y. N., Lammer H., Selsis F., Langmayr D., Jaritz G. F., Biernat H. K., 2007, A\&A, 472, 329
Espinoza N., Kossakowski D., Brahm R., 2018, preprint (arXiv:1812.08549) Espinoza N. et al., 2019, preprint (arXiv:1903.07694)

Essick R., Weinberg N. N., 2015, ApJ, 816, 18

Fabrycky D., Tremaine S., 2007, ApJ, 669, 1298

Fischer D. A., Valenti J., 2005, ApJ, 622, 1102

Ford E. B., Rasio F. A., 2008, ApJ, 686, 621

Ford E. B., Havlickova M., Rasio F. A., 2001, Icarus, 150, 303

Foreman-Mackey D., Hogg D. W., Lang D., Goodman J., 2013, PASP, 125, 306

Fulton B. J., Petigura E. A., Blunt S., Sinukoff E., 2018, PASP, 130, 044504 Gaia Collaboration, 2016, A\&A, 595, A2

Gaia Collaboration, 2018, A\&A, 616, A1

Gillen E., Hillenbrand L. A., David T. J., Aigrain S., Rebull L., Stauffer J., Cody A. M., Queloz D., 2017, ApJ, 849, 11

Gonzalez G., 1997, MNRAS, 285, 403

Guenther E. W. et al., 2017, A\&A, 608, A93

Günther M. N. et al., 2018, MNRAS, 478, 4720

Hebb L. et al., 2010, ApJ, 708, 224

Henden A., Munari U., 2014, Contrib. Astron. Obs. Skalnate Pleso, 43, 518

Husser T. O., Wende-von Berg S., Dreizler S., Homeier D., Reiners A., Barman T., Hauschildt P. H., 2013, A\&A, 553, A6

Irwin M. J. et al., 2004, in Quinn P. J., Bridger A., eds, Proc. SPIE Conf. Ser. Vol. 5493, Optimizing Scientific Return for Astronomy through Information Technologies. SPIE, Bellingham, p. 411

Jackson A. P., Davis T. A., Wheatley P. J., 2012, MNRAS, 422, 2024

Jackson B., Jensen E., Peacock S., Arras P., Penev K., 2016, Celest. Mech. Dyn. Astron., 126, 227

Jenkins J. S. et al., 2017, MNRAS, 466, 443

Kaufer A., Stahl O., Tubbesing S., Nørregaard P., Avila G., Francois P., Pasquini L., Pizzella A., 1999, The Messenger, 95, 8

Kovács G., Zucker S., Mazeh T., 2002, A\&A, 391, 369

Kreidberg L., 2015, PASP, 127, 1161

Li L. et al., 2018, Nat. Commun., 9, 3709

Lundkvist M. S. et al., 2016, Nat. Commun., 7, 11201

Maldonado J., Villaver E., Eiroa C., 2018, A\&A, 612, A93

Mandell A. M., Raymond S. N., Sigurdsson S., 2007, ApJ, 660, 823

Matsuo T., Shibai H., Ootsubo T., Tamura M., 2007, ApJ, 662, 1282

Mazeh T., Holczer T., Faigler S., 2016, A\&A, 589, A75

McCormac J. et al., 2017, PASP, 129, 025002

Mizuno H., 1980, Prog. Theor. Phys., 64, 544

Morton T. D., 2015, Astrophysics Source Code Library, record ascl: 1503.010

Murray-Clay R. A., Chiang E. I., Murray N., 2009, ApJ, 693, 23

Oberst T. E. et al., 2017, AJ, 153, 97

Owen J. E., Jackson A. P., 2012, MNRAS, 425, 2931

Owen J. E., Lai D., 2018, MNRAS, 479, 5012

Papaloizou J. C. B., Terquem C., 2001, MNRAS, 325, 221

Penev K. et al., 2016, AJ, 152, 127

Pepe F. et al., 2013, Nature, 503, 377

Petrovich C., Deibert E., Wu Y., 2019, AJ, 157, 180

Queloz D. et al., 2001a, A\&A, 379, 279

Queloz D. et al., 2001b, Messenger, 105, 1

Rasio F. A., Ford E. B., 1996, Science, 274, 954

Raynard L. et al., 2018, MNRAS, 481, 4960

Ricker G. R. et al., 2015, J. Astron. Telesc. Instrum. Syst., 1, 014003

Sanchis-Ojeda R., Rappaport S., Winn J. N., Kotson M. C., Levine A., El Mellah I., 2014, ApJ, 787, 47

Santerne A. et al., 2018, Nat. Astron., 2, 393

Santos N. C., Mayor M., Queloz D., Udry S., 2002, Messenger, 110, 32

Schlafly E. F., Finkbeiner D. P., 2011, ApJ, 737, 103

Skrutskie M. F. et al., 2006, AJ, 131, 1163

Soto M. G., Jenkins J. S., 2018, A\&A, 615, A76

Southworth J. et al., 2009, ApJ, 707, 167

Southworth J. et al., 2015, MNRAS, 447, 711

Stassun K. G. et al., 2018, AJ, 156, 102

Szabó G. M., Kiss L. L., 2011, ApJ, 727, L44

Tamuz O., Mazeh T., Zucker S., 2005, MNRAS, 356, 1466 
Terquem C., 2014, MNRAS, 444, 1738

Tonry J. L. et al., 2012, ApJ, 750, 99

Valsecchi F., Rasio F. A., Steffen J. H., 2014, ApJ, 793, L3

Wang J., Fischer D. A., 2015, AJ, 149, 14

Watson A. J., Donahue T. M., Walker J. C. G., 1981, Icarus, 48, 150

West R. G. et al., 2019, MNRAS, 486, 5094

Wheatley P. J. et al., 2013, in EPJ Web Conf., 47, 13002

Wheatley P. J. et al., 2018, MNRAS, 475, 4476

Winn J. N. et al., 2017, AJ, 154, 60

Winn J. N., Sanchis-Ojeda R., Rappaport S., 2018, New Astron. Rev., 83, 37

Wright E. L. et al., 2010, AJ, 140, 1868

Yee S. W., Petigura E. A., von Braun K., 2017, ApJ, 836, 77

Zacharias N., Finch C. T., Girard T. M., Henden A., Bartlett J. L., Monet D. G., Zacharias M. I., 2013, AJ, 145, 44

\section{SUPPORTING INFORMATION}

Supplementary data are available at MNRAS online.

Table 1. Photometry of NGTS, SAAO I, V and TESS for NGTS-6.

Please note: Oxford University Press is not responsible for the content or functionality of any supporting materials supplied by the authors. Any queries (other than missing material) should be directed to the corresponding author for the article.

This paper has been typeset from a $\mathrm{T}_{\mathrm{E}} \mathrm{X} / \mathrm{LT} \mathrm{E} \mathrm{X}$ file prepared by the author. 